\title{
铜催化的芳基炔酮的羟化三氟甲硫基化反应
}

\author{
胡娟娟 ${ }^{a} \quad$ 黄焰根 ${ }^{a}$ 徐修华 $b$ 卿凤翎*, $a, b$ \\ ( ${ }^{a}$ 东华大学化学化工与生物工程学院 生态纺织教育部重点实验室 上海 201620) \\ $\left({ }^{b}\right.$ 中国科学院上海有机化学研究所 有机氟化学重点实验室 上海 200032)
}

\begin{abstract}
摘要 近年来通过双官能团化的策略来制备含氟化合物已经成为氟化学研究的热点. 报道了一例铜催化的芳基炔酮的 差化一三氟甲硫基化反应来合成相应的含三氟甲硫基的烯醇. 铜盐和溶剂对于该反应产率的影响非常关键. 在最优条 件下, 一系列含三氟甲硫基的烯醇以中等到良好的产率被制备出来, 并且各种官能团能够在该反应中很好地兼容.

关键词 铜; 三氟甲硫基化; 羟化; 炔酮; 自由基
\end{abstract}

\section{Copper-Catalyzed Hydroxytrifluoromethylthiolation of Arylpropynones}

\author{
Hu, Juanjuan ${ }^{a} \quad$ Huang, Yangen $^{a} \quad \mathrm{Xu}$ Xiuhua $^{b} \quad$ Qing, Fengling ${ }^{*, a, b}$ \\ $\left({ }^{a}\right.$ Key Laboratory of Science and Technology of Eco-Textiles, Ministry of Education, College of Chemistry, \\ Chemical Engineering and Biotechnology, Donghua University, Shanghai 201620) \\ $\left({ }^{b}\right.$ Key Laboratory of Organofluorine Chemistry, Center for Excellence in Molecular Synthesis, Shanghai Institute of \\ Organic Chemistry, Chinese Academy of Sciences, Shanghai 200032)
}

\begin{abstract}
Recently, the preparation of fluorinated compounds through difunctionalization strategies has become a hot research area in fluorine chemistry. In this work, a copper-catalyzed hydroxytrifluoromethylthiolation of arylpropynones for the synthesis of the corresponding trifluoromethylthiolated enols was developed. The copper salt and solvent are crucial to the yields of this reaction. Under optimized reaction conditions, a series of trifluoromethylthiolated enols were obtained in moderate to good yields.
\end{abstract}

Keywords copper; trifluoromethylthiolation; hydroxylation; propynone; radical

\section{Introduction}

The incorporation of fluorine-containing groups into organic compounds can substantially change the chemical, physical, and biological properties. ${ }^{[1]}$ Among various fluorine-containing groups, the trifluoromethylthio group $\left(\mathrm{CF}_{3} \mathrm{~S}\right)$ has recently experienced a strong acceleration of interest because of its remarkable properties, in particular the strong electron-withdrawing effect and extremely high lipophilicity. ${ }^{[2]}$ Consequently, the development of new trifluoromethylthiolating reagents and new trifluoromethylthiolation reactions has provided sought-after strategies for the preparation of $\mathrm{SCF}_{3}$-containing compounds. ${ }^{[3]}$

Recently, difunctionalization reactions have become one of the most powerful strategies for the preparation of fluo- rinated compounds with simultaneous introduction of a fluorine or fluoroalkyl group with another functional group into organic molecules. This strategy has been widely applied in the synthesis of $\mathrm{SCF}_{3}$-containing compounds. ${ }^{[4 \sim 9]}$ For example, a series of trifluoromethylthiolated alkenes have been prepared by difunctionalization of alkynes. Cao and co-workers reported the anti-Markovnikov- and Markovnikov-selective hydrotrifluoromethylthiolation of terminal alkynes with $\mathrm{AgSCF}_{3}{ }^{[5]}$ Recently, $\mathrm{Liu}^{[6 \mathrm{a}]}$ and our group $^{[6 b]}$ respectively disclosed the tandem trifluoromethylthiolation/aryl migration of aryl propynyl ethers or aryl alkynoates for the preparation of corresponding trifluoromethylthiolated alkenes. The stereoselective bis-trifluoromethylthiolation of alkynes was reported by Tlili and Billard $^{[7 \mathrm{a}]}$ as well as by our group. ${ }^{[7 \mathrm{~b}]}$ Very recently, Yi and

\footnotetext{
* Corresponding author. E-mail: flq@mai.sioc.ac.cn

Received August 30, 2018; revised October 23, 2018; published online November 11, 2018

Dedicated to Professor Qingyun Chen on the occasion of his 90th birthday.

Project supported by the National Natural Science Foundation of China (Nos. 21332010, 21421002), the Strategic Priority Research Program of the Chinese Academy of Sciences (No. XDB20000000), and the Youth Innovation Promotion Association of the Chinese Academy of Sciences (No. 2016234).

国家自然科学基金(Nos. 21332010, 21421002)、中国科学院战略性先导科技专项(No. XDB20000000)和中国科学院青年创新促进会(No. 2016234)资助 项目.
} 
Zhang et al. ${ }^{[8]}$ developed a chlorotrifluoromethylthiolation of alkynes using $\mathrm{CF}_{3} \mathrm{SO}_{2} \mathrm{Cl}$ as a bifunctionalization reagent.

Oxytrifluoromethylthiolation of alkynes is a useful bifunctionalization reaction because $O$-containing groups $\left(\mathrm{OSO}_{2} \mathrm{R}, \mathrm{OCOR}, \mathrm{OH}\right.$, etc. $)$ could be readily transformed to other functional groups. The Billard group ${ }^{[9 \mathrm{a}]}$ first reported the oxytrifluoromethylthiolation of alkynes with an electrophilic trifluoromethylthiolating reagent under $\mathrm{BF}_{3} \bullet \mathrm{Et}_{2} \mathrm{O}$ activation (Scheme 1a). Recently, Zhao et al. ${ }^{[9 b]}$ disclosed a selenide-catalyzed regio- and stereo-selective difunctionalization of alkynes to afford trifluoromethylthiolated alkenyl triflates (Scheme 1b). However, these electrophilic processes are limited to electron-rich alkynes. To the best of our knowledge, the oxytrifluoromethylthiolation of alkynes bearing electron-withdrawing substituents remains underexplored. To extend the substrate scope of oxytrifluoromethylthiolation, and as part of our continued interest in the difunctionalization reactions, ${ }^{[4 \mathrm{~b}, \mathrm{~m}, 6 \mathrm{~b}, 7 \mathrm{~b}, 10]}$ herein we disclose a copper-catalyzed oxidative hydroxytrifluoromethylthiolation of arylpropynones with $\mathrm{AgSCF}_{3}$ (Scheme 1c).

$$
\mathrm{R}_{\mathrm{R}^{1}, \mathrm{R}^{2}=\text { alkyl, } \mathrm{H}}^{\text {previous work }} \stackrel{\mathrm{TsONa}}{\mathrm{BF}_{3} \cdot \mathrm{Et}_{2} \mathrm{O}}
$$

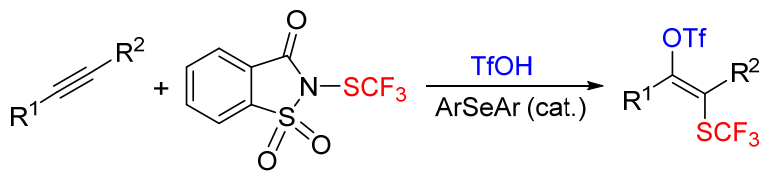

$$
\mathrm{R}^{1}, \mathrm{R}^{2}=\text { aryl, alkyl, } \mathrm{H}
$$

this work

$$
\mathrm{Ar}_{\mathrm{R}} \mathrm{AgSCF}_{3} \underset{\text { oxidant }}{\stackrel{\mathrm{Cu} \text { cat. }}{\mathrm{H}_{2} \mathrm{O}}} \overbrace{\mathrm{SCF}_{3}}^{\mathrm{OH}}
$$

Scheme 1 Oxytrifluoromethylthiolation of alkynes

\section{Results and discussion}

Our initial attempt began by employing 4-phenylbut3-yn-2-one (1a) as the model substrate with $\mathrm{AgSCF}_{3}$ and $\mathrm{H}_{2} \mathrm{O}$ as the $\mathrm{SCF}_{3}$ and $\mathrm{OH}$ sources, respectively (Table 1). However, the reaction in the presence of $\left(\mathrm{NH}_{4}\right)_{2} \mathrm{~S}_{2} \mathrm{O}_{8}$ in $\mathrm{MeCN}$ could not yield the desired trifluoromethylthiolated enol 2a (Entry 1). To our delight, 2a was formed in 32\% yield when catalytic $\mathrm{Cu}(\mathrm{OAc})_{2}$ was added to the reaction mixture (Entry 2). The screening of oxidants demonstrated that original $\left(\mathrm{NH}_{4}\right)_{2} \mathrm{~S}_{2} \mathrm{O}_{8}$ was the best of choice (Entries $3 \sim 5$ ). Subsequently, a series of $\mathrm{Cu}$ salts including $\mathrm{CuCl}_{2}$, $\mathrm{CuF}_{2}, \mathrm{CuSO}_{4}, \mathrm{CuCl}, \mathrm{Cu}(\mathrm{OTf})_{2}, \mathrm{Cu}(\mathrm{TFA})_{2}$, and $\mathrm{CuCN}$ were investigated (Entries $6 \sim 12$ ), but none of them gave better result. The addition of catalytic monodentate or bidentate ligand was found to be beneficial for the yield (Entries
$13 \sim 19)$. Among the ligands $\mathbf{L} \mathbf{1} \sim \mathbf{L} 7$ tested, 4,4'-dimethyl2,2'-bipyridine (dmbpy, L3) was optimal, affording 2a in $46 \%$ yield (Entry 15 ). Finally, the effect of solvent on the reaction was examined. Switching $\mathrm{MeCN}$ to THF, DMF, or $\mathrm{MeCN} / \mathrm{H}_{2} \mathrm{O}$ resulted in much lower yields (Entries 20 22). The use of $\mathrm{HOAc}$ or $\mathrm{HCO}_{2} \mathrm{H}$ as a co-solvent could sharply improve the yields (Entries 23 and 24), whereas slightly lower yield was obtained when the reaction was

\begin{tabular}{|c|c|c|c|c|}
\hline Entry & Oxidant & Additive & Solvent & Yield $^{b} / \%$ \\
\hline 1 & $\left(\mathrm{NH}_{4}\right)_{2} \mathrm{~S}_{2} \mathrm{O}_{8}$ & - & $\mathrm{MeCN}$ & 0 \\
\hline 2 & $\left(\mathrm{NH}_{4}\right)_{2} \mathrm{~S}_{2} \mathrm{O}_{8}$ & $\mathrm{Cu}(\mathrm{OAc})_{2}$ & $\mathrm{MeCN}$ & 32 \\
\hline 3 & $\mathrm{Na}_{2} \mathrm{~S}_{2} \mathrm{O}_{8}$ & $\mathrm{Cu}(\mathrm{OAc})_{2}$ & $\mathrm{MeCN}$ & 29 \\
\hline 4 & $\mathrm{~K}_{2} \mathrm{~S}_{2} \mathrm{O}_{8}$ & $\mathrm{Cu}(\mathrm{OAc})_{2}$ & $\mathrm{MeCN}$ & 22 \\
\hline 5 & ТВНР & $\mathrm{Cu}(\mathrm{OAc})_{2}$ & $\mathrm{MeCN}$ & Trace \\
\hline 6 & $\left(\mathrm{NH}_{4}\right)_{2} \mathrm{~S}_{2} \mathrm{O}_{8}$ & $\mathrm{CuCl}_{2}$ & $\mathrm{MeCN}$ & 24 \\
\hline 7 & $\left(\mathrm{NH}_{4}\right)_{2} \mathrm{~S}_{2} \mathrm{O}_{8}$ & $\mathrm{CuF}_{2}$ & $\mathrm{MeCN}$ & 27 \\
\hline 8 & $\left(\mathrm{NH}_{4}\right)_{2} \mathrm{~S}_{2} \mathrm{O}_{8}$ & $\mathrm{CuSO}_{4}$ & $\mathrm{MeCN}$ & 15 \\
\hline 9 & $\left(\mathrm{NH}_{4}\right)_{2} \mathrm{~S}_{2} \mathrm{O}_{8}$ & $\mathrm{Cu}(\mathrm{OTf})_{2}$ & $\mathrm{MeCN}$ & 19 \\
\hline 10 & $\left(\mathrm{NH}_{4}\right)_{2} \mathrm{~S}_{2} \mathrm{O}_{8}$ & $\mathrm{Cu}(\mathrm{TFA})_{2}$ & $\mathrm{MeCN}$ & 11 \\
\hline 11 & $\left(\mathrm{NH}_{4}\right)_{2} \mathrm{~S}_{2} \mathrm{O}_{8}$ & $\mathrm{CuCI}$ & $\mathrm{MeCN}$ & 24 \\
\hline 12 & $\left(\mathrm{NH}_{4}\right)_{2} \mathrm{~S}_{2} \mathrm{O}_{8}$ & $\mathrm{CuCN}$ & $\mathrm{MeCN}$ & 19 \\
\hline 13 & $\left(\mathrm{NH}_{4}\right)_{2} \mathrm{~S}_{2} \mathrm{O}_{8}$ & $\mathrm{Cu}(\mathrm{OAc})_{2} / \mathbf{L 1}$ & $\mathrm{MeCN}$ & 38 \\
\hline 14 & $\left(\mathrm{NH}_{4}\right)_{2} \mathrm{~S}_{2} \mathrm{O}_{8}$ & $\mathrm{Cu}(\mathrm{OAc})_{2} / \mathbf{L} \mathbf{2}$ & $\mathrm{MeCN}$ & 35 \\
\hline 15 & $\left(\mathrm{NH}_{4}\right)_{2} \mathrm{~S}_{2} \mathrm{O}_{8}$ & $\mathrm{Cu}(\mathrm{OAc})_{2} / \mathbf{L} \mathbf{3}$ & $\mathrm{MeCN}$ & 46 \\
\hline 16 & $\left(\mathrm{NH}_{4}\right)_{2} \mathrm{~S}_{2} \mathrm{O}_{8}$ & $\mathrm{Cu}(\mathrm{OAc})_{2} / \mathbf{L} \mathbf{4}$ & $\mathrm{MeCN}$ & 41 \\
\hline 17 & $\left(\mathrm{NH}_{4}\right)_{2} \mathrm{~S}_{2} \mathrm{O}_{8}$ & $\mathrm{Cu}(\mathrm{OAc})_{2} / \mathbf{L 5}$ & $\mathrm{MeCN}$ & 33 \\
\hline 18 & $\left(\mathrm{NH}_{4}\right)_{2} \mathrm{~S}_{2} \mathrm{O}_{8}$ & $\mathrm{Cu}(\mathrm{OAc})_{2} / \mathbf{L 6}$ & $\mathrm{MeCN}$ & 40 \\
\hline 19 & $\left(\mathrm{NH}_{4}\right)_{2} \mathrm{~S}_{2} \mathrm{O}_{8}$ & $\mathrm{Cu}(\mathrm{OAc})_{2} / \mathbf{L} 7$ & $\mathrm{MeCN}$ & 34 \\
\hline 20 & $\left(\mathrm{NH}_{4}\right)_{2} \mathrm{~S}_{2} \mathrm{O}_{8}$ & $\mathrm{Cu}(\mathrm{OAc})_{2} / \mathbf{L} 3$ & THF & 13 \\
\hline 21 & $\left(\mathrm{NH}_{4}\right)_{2} \mathrm{~S}_{2} \mathrm{O}_{8}$ & $\mathrm{Cu}(\mathrm{OAc})_{2} / \mathbf{L} \mathbf{3}$ & DMF & 19 \\
\hline $22^{c}$ & $\left(\mathrm{NH}_{4}\right)_{2} \mathrm{~S}_{2} \mathrm{O}_{8}$ & $\mathrm{Cu}(\mathrm{OAc})_{2} / \mathbf{L} \mathbf{3}$ & $\mathrm{MeCN} / \mathrm{H}_{2} \mathrm{O}$ & 8 \\
\hline $23^{c}$ & $\left(\mathrm{NH}_{4}\right)_{2} \mathrm{~S}_{2} \mathrm{O}_{8}$ & $\mathrm{Cu}(\mathrm{OAc})_{2} / \mathbf{L} 3$ & $\mathrm{MeCN} / \mathrm{HOAc}$ & 68 \\
\hline $24^{c}$ & $\left(\mathrm{NH}_{4}\right)_{2} \mathrm{~S}_{2} \mathrm{O}_{8}$ & $\mathrm{Cu}(\mathrm{OAc})_{2} / \mathbf{L} 3$ & $\mathrm{MeCN} / \mathrm{HCO}_{2} \mathrm{H}$ & 89 \\
\hline $25^{c}$ & $\left(\mathrm{NH}_{4}\right)_{2} \mathrm{~S}_{2} \mathrm{O}_{8}$ & $\mathrm{Cu}(\mathrm{OAc})_{2} / \mathbf{L 3}$ & $\mathrm{MeCN} / \mathrm{TFA}$ & 35 \\
\hline
\end{tabular}
performed in MeCN/TFA (Entry 25).

Table 1 Optimization of reaction conditions ${ }^{a}$

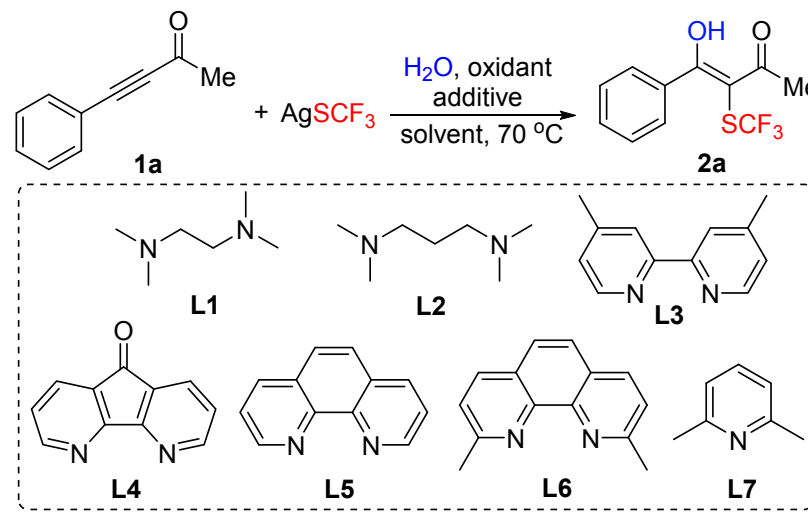

${ }^{a}$ Reaction conditions: 1a $(0.1 \mathrm{mmol}), \mathrm{AgSCF}_{3}(0.2 \mathrm{mmol}), \mathrm{H}_{2} \mathrm{O}(0.3 \mathrm{mmol})$, oxidant $(0.2 \mathrm{mmol})$, additive $(0.02 \mathrm{mmol})$, solvent $(2.0 \mathrm{~mL})$, under $\mathrm{N}_{2}, 70{ }^{\circ} \mathrm{C}$, 3 h. ${ }^{b}$ Yields determined by ${ }^{19} \mathrm{~F}$ NMR spectroscopy using trifluoromethybenzene as an internal standard. ${ }^{c} \mathrm{MeCN} / \mathrm{co}-$ solvent $(1.0 \mathrm{~mL} / 1.0 \mathrm{~mL})$.

With the optimized reaction conditions in hand (Table 1, Entry 19), the scope and limitation of hydroxytrifluoromethylthiolation of arylpropynones were studied. As shown in 
Table 2, a series of 4-arylbut-3-yn-2-ones $(\mathbf{1 a} \sim \mathbf{1 0})$ were converted to the corresponding trifluoromethylthiolated enols in moderate to high yields. Notably, in all cases, traces amounts of trifluoromethylthiolated 1,3-diketones could be detected. This reaction exhibited good functional group compatibility, and different functional groups such as methoxy, fluoro, chloro, nitro, cyano, and trifluoromethyl were well tolerated. Furthermore, the electronic and steric effect of substituent groups exerted a clear influence on this transformation. In general, ortho-substituted (1k) and meta-substituted (1c) substrates distinctly affected the yields, and substrates with electron-deficient moieties (1n, 10) gave much lower yields than those with electron-rich moieties $(\mathbf{1 b}, \mathbf{1 f})$. Gratifyingly, this transformation proceeded smoothly for the substrates with a naphthyl group (1m) or a heterocyclic group (2-thienyl, 1p). The final concern was that 1-phenylpent-1-yn-3-one (1q) also showed good compatibility in this reaction system. Notably, when diaryl propynones $\left(\mathrm{R}^{2}=\right.$ aryl) were subjected to the standard conditions, cascade trifluoromethylthiolation and cyclization happened affording trifluoromethylthiolated indenones as the major products. ${ }^{[11 i]}$

On the basis of previous reports, a plausible reaction mechanism was proposed (Scheme 2). Initially, $\mathrm{AgSCF}_{3}$ is oxidized by $\left(\mathrm{NH}_{4}\right)_{2} \mathrm{~S}_{2} \mathrm{O}_{8}$ to furnish the $\mathrm{SCF}_{3}$ radical. ${ }^{[5,11]}$ Then, the addition of $\mathrm{SCF}_{3}$ radical to arylpropynones generates radical intermediate $\mathbf{A}$, which is then oxidized to cation intermediate $\mathbf{B}$ by $\mathrm{Cu} /\left(\mathrm{NH}_{4}\right)_{2} \mathrm{~S}_{2} \mathrm{O}_{8}$ (path A). Finally, the attack of cation $\mathbf{B}$ with $\mathrm{H}_{2} \mathrm{O}$ furnishes the desired product 2 . However, an alternative pathway involving the formation of $\mathrm{Cu}^{\mathrm{III}}$-complex $\mathbf{C}^{[12]}$ can not be excluded (path B).

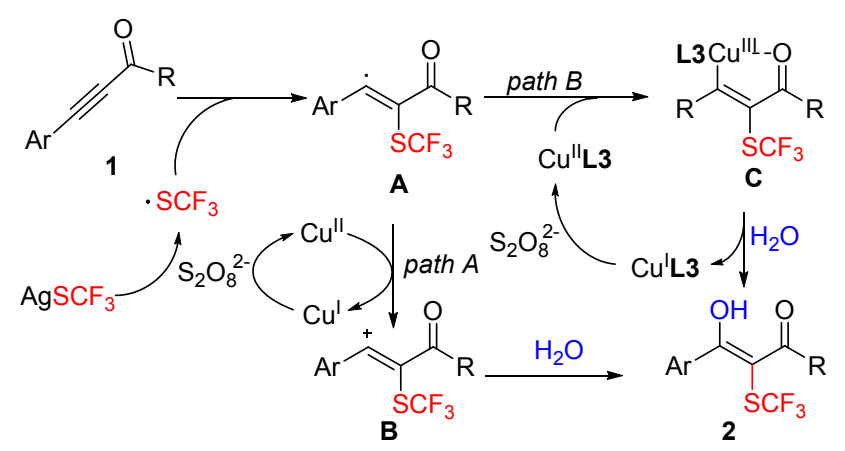

Scheme 2 Proposed reaction mechanism

\section{Conclusions}

In conclusion, we have developed a copper-catalyzed hydroxytrifluoromethylthiolation of arylpropynones with $\mathrm{AgSCF}_{3}$ and $\mathrm{H}_{2} \mathrm{O}$ as the $\mathrm{SCF}_{3}$ and $\mathrm{OH}$ sources, respectively. This work represents the first example of oxytrifluoromethylthiolation of alkynes bearing electron-withdrawing substituents. A series of trifluoromethylthiolated enols were obtained in moderate to high yields. We believe that this protocol will find applications in the preparation of useful building blocks and bio-relevant molecules.
Table 2 Hydroxytrifluoromethylthiolation of arylpropynones ${ }^{a}$

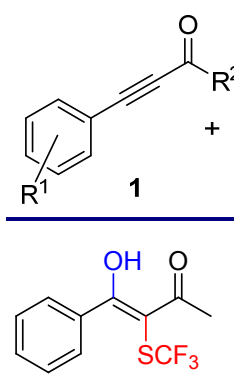

2a, $78 \%$<smiles>CC(=O)C(=C(O)c1ccc(C)cc1)C(F)(F)F</smiles>

2b, $72 \%$

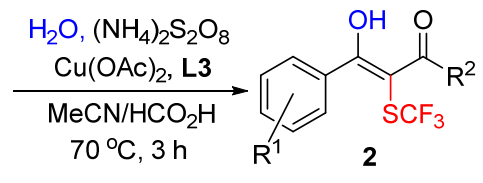<smiles>CC(=O)C(=C(O)c1cccc(C)c1)C(F)(F)F</smiles>

2c, $44 \%$<smiles>CCCCc1ccc(C(O)=C(C(C)=O)C(F)(F)F)cc1</smiles>

2d, $59 \%$

2e, $52 \%$

2f, $66 \%$<smiles>CC(=O)/C(=C(/O)c1ccc(F)cc1)C(F)(F)F</smiles>

2g, $51 \%$<smiles>CC(=O)C(SC(C)(F)F)=C(O)c1cccc(F)c1</smiles>

2h, $53 \%$<smiles>[R20]C(F)(F)[Si](C(C)=O)=C(O)c1ccc(Cl)cc1</smiles>

2i, $60 \%$<smiles>CC(=O)C(=C(O)c1cccc(Cl)c1)C(F)(F)F</smiles><smiles>O=C(CC(=O)C(F)(F)F)C(O)c1ccccc1Cl</smiles>

2k, 37\%<smiles>CC(=O)C(=C(O)c1ccc(C(F)(F)F)cc1)C(F)(F)F</smiles>

2I, $41 \%$<smiles>CC(=O)/C([Se-]C(F)(F)F)=C(/O)c1cccc2ccccc12</smiles>

2m, 73\%<smiles>CC(=O)C(=C(O)c1ccc(C#N)cc1)C(F)(F)F</smiles>

2n, $36 \%$<smiles>CC(=O)C(=C(O)c1ccc([N+](=O)[O-])cc1)C(F)(F)F</smiles>

2o, $26 \%$<smiles>CCC(=O)C(=C(O)c1cccs1)C(F)(F)F</smiles>

2p, $57 \%$<smiles>CCC(=O)/C(=C(/O)c1ccccc1)C(F)(F)F</smiles>

2q, $61 \%$

${ }^{a}$ Reaction conditions: $1(0.5 \mathrm{mmol}), \mathrm{AgSCF}_{3}(1.0 \mathrm{mmol}), \mathrm{H}_{2} \mathrm{O}(1.5 \mathrm{mmol})$, $\left(\mathrm{NH}_{4}\right)_{2} \mathrm{~S}_{2} \mathrm{O}_{8}(1.0 \mathrm{mmol}), \mathrm{Cu}(\mathrm{OAc})_{2}(0.1 \mathrm{mmol}), \mathbf{L 3}(0.1 \mathrm{mmol}), \mathrm{MeCN} /$ $\mathrm{HCO}_{2} \mathrm{H}(5.0 \mathrm{~mL} / 5.0 \mathrm{~mL})$, under $\mathrm{N}_{2}, 70{ }^{\circ} \mathrm{C}, 3 \mathrm{~h}$, isolated yields.

\section{Experimental section}

\subsection{General information}

${ }^{1} \mathrm{H}$ NMR and ${ }^{19} \mathrm{~F}$ NMR $\left(\mathrm{CFCl}_{3}\right.$ as outside standard and low field is positive) spectra were recorded on a Bruker AM 400 spectrometer. ${ }^{13} \mathrm{C}$ NMR spectra were recorded on a Bruker AM 400 spectrometer. High resolution mass spectra (HRMS) were performed using a GC/MS TOF high-resolution mass spectrometer equipped with a liquid chromatography system. IR spectra were recorded on a Thermo Scientific Nicolet 380 FT-IR spectrometer.

4.2 General procedure for hydroxytrifluoromethylthiolation of arylpropynones

A mixture of arylpropynone $1(0.50 \mathrm{mmol}), \mathrm{AgSCF}_{3}$ (208.9 mg, $1.0 \mathrm{mmol}), \mathrm{H}_{2} \mathrm{O}(27 \mu \mathrm{L}, 1.5 \mathrm{mmol}),\left(\mathrm{NH}_{4}\right)_{2^{-}}$ 
$\mathrm{S}_{2} \mathrm{O}_{8}(228.2 \mathrm{mg}, 1.0 \mathrm{mmol}), \mathrm{Cu}(\mathrm{OAc})_{2}(18.2 \mathrm{mg}, 0.1$ $\mathrm{mmol}$ ), and 4,4'-dimethyl-2,2'-bipyridine (18.4 $\mathrm{mg}, 0.1$ mmol) was added tube that was sealed with a septum. The mixture was evacuated and backfilled with nitrogen three times. $\mathrm{MeCN}(5.0 \mathrm{~mL})$ and $\mathrm{HCO}_{2} \mathrm{H}(5.0 \mathrm{~mL})$ were added to the tube. Then, the tube was stirred at $70{ }^{\circ} \mathrm{C}$ for $3 \mathrm{~h}$. After the reaction was complete, saturated $\mathrm{NH}_{4} \mathrm{Cl}$ solution was added. The resulting mixture was extracted with EtOAc for three times. The combined organic layer was dried over anhydrous $\mathrm{Na}_{2} \mathrm{SO}_{4}$ and concentrated under reduced pressure. The residue was purified by silica gel column chromatography to give product 2 .

(E)-4-Hydroxy-4-phenyl-3-(trifluoromethylthio)but-3en-2-one $(\mathbf{2 a})^{[13]}$ : yellow oil, $102.7 \mathrm{mg}, 78 \%$ yield. ${ }^{1} \mathrm{H}$ NMR (400 MHz, $\left.\mathrm{CDCl}_{3}\right) \delta: 7.56 \sim 7.51(\mathrm{~m}, 2 \mathrm{H}), 7.44 \sim$ $7.38(\mathrm{~m}, 1 \mathrm{H}), 7.38 \sim 7.32(\mathrm{~m}, 2 \mathrm{H}), 2.48(\mathrm{~s}, 3 \mathrm{H}) ;{ }^{13} \mathrm{C}$ NMR $\left(101 \mathrm{MHz}, \mathrm{CDCl}_{3}\right) \delta: 201.7,195.0,135.7,127.9,131.3$, $129.1(\mathrm{q}, J=312.1 \mathrm{~Hz}), 128.7,95.4,25.2 ;{ }^{19} \mathrm{~F}$ NMR $(376$ $\left.\mathrm{MHz}, \mathrm{CDCl}_{3}\right) \delta:-45.85(\mathrm{~s}, 3 \mathrm{~F})$.

(E)-4-Hydroxy-4-p-tolyl-3-(trifluoromethylthio)but-3en-2-one (2b): yellow oil, $99.2 \mathrm{mg}, 72 \%$ yield. ${ }^{1} \mathrm{H}$ NMR $\left(400 \mathrm{MHz}, \mathrm{CDCl}_{3}\right) \delta: 7.48(\mathrm{~d}, J=8.2 \mathrm{~Hz}, 2 \mathrm{H}), 7.16(\mathrm{~d}, J=$ $7.9 \mathrm{~Hz}, 2 \mathrm{H}), 2.48$ (s, 3H), $2.33(\mathrm{~s}, 3 \mathrm{H}) ;{ }^{13} \mathrm{C}$ NMR (101 $\left.\mathrm{MHz}, \mathrm{CDCl}_{3}\right) \delta: 200.6,193.7,141.0,131.8,128.1$ (q, $J=$ $312.1 \mathrm{~Hz}), 127.9,127.6,94.0,24.1,20.5 ;{ }^{19} \mathrm{~F}$ NMR (376 $\mathrm{MHz}, \mathrm{CDCl}_{3}$ ) $\delta:-45.89$ (s, 3F); IR (film) v: 3678, 2933, 1609, 1397, 1100, 825, 750, $565 \mathrm{~cm}^{-1}$; MS (ESI) $\mathrm{m} / \mathrm{z}: 277$ $[\mathrm{M}+\mathrm{H}]^{+}$; HRMS (ESI-TOF) calcd for $\mathrm{C}_{12} \mathrm{H}_{12} \mathrm{~F}_{3} \mathrm{O}_{2} \mathrm{~S}[\mathrm{M}+$ $\mathrm{H}]^{+}:$277.0505, found 277.0506.

(E)-4-Hydroxy-4- $m$-tolyl-3-(trifluoromethylthio)but-3en-2-one (2c): yellow oil, $61.0 \mathrm{mg}, 44 \%$ yield. ${ }^{1} \mathrm{H}$ NMR $\left(400 \mathrm{MHz}, \mathrm{CDCl}_{3}\right) \delta: 7.30(\mathrm{~s}, 2 \mathrm{H}), 7.19 \sim 7.18(\mathrm{~m}, 2 \mathrm{H})$, $2.44(\mathrm{~s}, 3 \mathrm{H}), 2.27(\mathrm{~s}, 3 \mathrm{H}) ;{ }^{13} \mathrm{C}$ NMR $\left(101 \mathrm{MHz}, \mathrm{CDCl}_{3}\right) \delta$ : 200.6, 194.2, 136.7, 134.6, 131.0, 128.2 (q, $J=312.1 \mathrm{~Hz}$ ), 128.1, 126.7, 124.8, 94.3, 24.2, 20.3; ${ }^{19} \mathrm{~F}$ NMR (376 MHz, $\left.\mathrm{CDCl}_{3}\right) \delta:-45.84(\mathrm{~s}, 3 \mathrm{~F})$; IR (film) $v: 3661,2987,1695$, 1409, 1107, $738 \mathrm{~cm}^{-1}$; MS (ESI) $m / z: 277[\mathrm{M}+\mathrm{H}]^{+}$; HRMS (ESI-TOF) calcd for $\mathrm{C}_{12} \mathrm{H}_{12} \mathrm{~F}_{3} \mathrm{O}_{2} \mathrm{~S}[\mathrm{M}+\mathrm{H}]^{+}$: 277.0505, found 277.0503.

(E)-4-(4-Ethylphenyl)-4-hydroxy-3-(trifluoromethylthio)but-3-en-2-one (2d): yellow oil, $85.9 \mathrm{mg}, 59 \%$ yield. ${ }^{1} \mathrm{H}$ NMR (400 MHz, $\left.\mathrm{CDCl}_{3}\right) \delta: 7.51(\mathrm{~d}, J=8.2 \mathrm{~Hz}, 2 \mathrm{H}), 7.18$ (d, $J=8.2 \mathrm{~Hz}, 2 \mathrm{H}), 2.63(\mathrm{q}, J=7.6 \mathrm{~Hz}, 2 \mathrm{H}), 2.49(\mathrm{~s}, 3 \mathrm{H})$, $1.19(\mathrm{t}, J=7.6 \mathrm{~Hz}, 3 \mathrm{H}) ;{ }^{13} \mathrm{C}$ NMR $\left(101 \mathrm{MHz}, \mathrm{CDCl}_{3}\right) \delta$ : 201.7, 194.6, 148.2, 133.0, 129.2 (q, $J=312.1 \mathrm{~Hz}), 129.1$, 127.4, 95.1, 28.9, 25.3, 15.1; ${ }^{19} \mathrm{~F}$ NMR (376 MHz, $\left.\mathrm{CDCl}_{3}\right)$ $\delta:-45.87(\mathrm{~s}, 3 \mathrm{~F})$; IR (film) v: 3676, 2969, 1609, 1397 , 1102, 1018, 838, $751 \mathrm{~cm}^{-1}$; MS (ESI) $\mathrm{m} / z: 291[\mathrm{M}+\mathrm{H}]^{+}$; HRMS (ESI-TOF) calcd for $\mathrm{C}_{13} \mathrm{H}_{14} \mathrm{~F}_{3} \mathrm{O}_{2} \mathrm{~S}[\mathrm{M}+\mathrm{H}]^{+}$: 291.0661, found 291.0662.

(E)-4-(4-Butylphenyl)-4-hydroxy-3-(trifluoromethylthio)but-3-en-2-one (2e): yellow oil, $83.2 \mathrm{mg}, 52 \%$ yield. ${ }^{1} \mathrm{H}$ NMR (400 MHz, $\left.\mathrm{CDCl}_{3}\right) \delta: 7.50(\mathrm{~d}, J=8.2 \mathrm{~Hz}, 2 \mathrm{H}), 7.16$ $(\mathrm{d}, J=7.9 \mathrm{~Hz}, 2 \mathrm{H}), 2.58(\mathrm{t}, J=8.0 \mathrm{~Hz}, 2 \mathrm{H}), 2.48(\mathrm{~s}, 3 \mathrm{H})$, $1.57 \sim 1.51(\mathrm{~m}, 2 \mathrm{H}), 1.31 \sim 1.28(\mathrm{~m}, 2 \mathrm{H}), 0.85(\mathrm{t}, J=7.2$ $\mathrm{Hz}, 3 \mathrm{H}) ;{ }^{13} \mathrm{C}$ NMR $\left(101 \mathrm{MHz}, \mathrm{CDCl}_{3}\right) \delta: 200.6,193.6$, 145.9, 131.9, 128.2 (q, $J=312.1 \mathrm{~Hz}), 128.0,126.9,94.0$,
34.6, 32.2, 24.3, 21.3, 12.9; ${ }^{19} \mathrm{~F}$ NMR $\left(376 \mathrm{MHz}, \mathrm{CDCl}_{3}\right) \delta$ : -45.87 (s, 3F); IR (film) v: 3673, 2960, 1681, 1603, 1403, 1101, $747 \mathrm{~cm}^{-1}$; MS (ESI) m/z: $319[\mathrm{M}+\mathrm{H}]^{+}$; HRMS (ESI-TOF) calcd for $\mathrm{C}_{15} \mathrm{H}_{18} \mathrm{~F}_{3} \mathrm{O}_{2} \mathrm{~S}[\mathrm{M}+\mathrm{H}]^{+}: 319.0974$, found 319.0973 .

(E)-4-Hydroxy-4-(4-methoxyphenyl)-3-(trifluoromethylthio)but-3-en-2-one (2f): yellow oil, $96.9 \mathrm{mg}, 66 \%$ yield. ${ }^{1} \mathrm{H}$ NMR (400 MHz, $\left.\mathrm{CDCl}_{3}\right) \delta: 7.65(\mathrm{~d}, J=8.9 \mathrm{~Hz}, 2 \mathrm{H})$, $6.86(\mathrm{~d}, J=8.9 \mathrm{~Hz}, 2 \mathrm{H}), 3.80(\mathrm{~s}, 3 \mathrm{H}), 2.49(\mathrm{~s}, 3 \mathrm{H}) ;{ }^{13} \mathrm{C}$ NMR (101 MHz, $\left.\mathrm{CDCl}_{3}\right) \delta:$ 200.4, 192.5, 161.3, 130.4, 128.2 (q, $J=313.1 \mathrm{~Hz}), 126.8,112.2,93.5,54.4,24.3 ;{ }^{19} \mathrm{~F}$ NMR (376 MHz, $\left.\mathrm{CDCl}_{3}\right) \delta$ : -45.97 (s, 3F); IR (film) $v$ : 3668, 2965, 1602, 1253, 1100, 837, $752 \mathrm{~cm}^{-1}$; MS (ESI) $m / z: 293[\mathrm{M}+\mathrm{H}]^{+}$; HRMS (ESI-TOF) calcd for $\mathrm{C}_{12} \mathrm{H}_{12} \mathrm{~F}_{3} \mathrm{O}_{3} \mathrm{~S}[\mathrm{M}+\mathrm{H}]^{+}: 293.0454$, found 293.0455.

(E)-4-(4-Fluorophenyl)-4-hydroxy-3-(trifluoromethylthio)but-3-en-2-one (2g): yellow oil, $71.7 \mathrm{mg}, 51 \%$ yield. ${ }^{1} \mathrm{H}$ NMR $\left(400 \mathrm{MHz}, \mathrm{CDCl}_{3}\right) \delta: 7.62 \sim 7.58(\mathrm{~m}, 2 \mathrm{H}), 7.05$ $(\mathrm{t}, J=8.6 \mathrm{~Hz}, 2 \mathrm{H}), 2.49(\mathrm{~s}, 3 \mathrm{H}) ;{ }^{13} \mathrm{C} \mathrm{NMR}(101 \mathrm{MHz}$, $\left.\mathrm{CDCl}_{3}\right) \delta: 200.5,192.8,163.4(\mathrm{~d}, J=251.5 \mathrm{~Hz}), 130.8(\mathrm{~d}$, $J=4.0 \mathrm{~Hz}), 130.3(\mathrm{~d}, J=9.1 \mathrm{~Hz}), 128.0(\mathrm{q}, J=312.1 \mathrm{~Hz})$, $114.1(\mathrm{~d}, J=22.2 \mathrm{~Hz}), 94.1,24.1 ;{ }^{19} \mathrm{~F}$ NMR $(376 \mathrm{MHz}$, $\left.\mathrm{CDCl}_{3}\right) \delta:-45.91(\mathrm{~s}, 3 \mathrm{~F}),-107.30(\mathrm{~s}, 1 \mathrm{~F})$; IR (film) $v$ : 3667, 2984, 1603, 1406, 1104, 841, $748 \mathrm{~cm}^{-1}$; MS (ESI) $m / z: 281[\mathrm{M}+\mathrm{H}]^{+}$; HRMS (ESI-TOF) calcd for $\mathrm{C}_{11} \mathrm{H}_{9} \mathrm{~F}_{4} \mathrm{O}_{2} \mathrm{~S}[\mathrm{M}+\mathrm{H}]^{+}: 281.0254$, found 281.0254 .

(E)-4-(3-Fluorophenyl)-4-hydroxy-3-(trifluoromethylthio)but-3-en-2-one (2h): yellow oil, $74.5 \mathrm{mg}, 53 \%$ yield. ${ }^{1} \mathrm{H}$ NMR $\left(400 \mathrm{MHz}, \mathrm{CDCl}_{3}\right) \delta: 7.32 \sim 7.31(\mathrm{~m}, 2 \mathrm{H})$, $7.25 \sim 7.22(\mathrm{~m}, 1 \mathrm{H}), 7.13 \sim 7.09(\mathrm{~m}, 1 \mathrm{H}), 2.48(\mathrm{~s}, 3 \mathrm{H}) ;{ }^{13} \mathrm{C}$ NMR (101 MHz, $\left.\mathrm{CDCl}_{3}\right) \delta: 201.5,193.9,162.0$ (d, $J=$ $248.5 \mathrm{~Hz}), 137.7$ (d, $J=7.1 \mathrm{~Hz}), 129.6$ (d, $J=8.1 \mathrm{~Hz})$, 129.0 (q, $J=312.1 \mathrm{~Hz}), 124.4$ (d, $J=3.0 \mathrm{~Hz}), 118.2$ (d, $J=21.2 \mathrm{~Hz}), 115.8(\mathrm{~d}, J=23.2 \mathrm{~Hz}), 95.5,25.0 ;{ }^{19} \mathrm{~F}$ NMR $\left(376 \mathrm{MHz}, \mathrm{CDCl}_{3}\right) \delta:-45.80(\mathrm{~s}, 3 \mathrm{~F}),-112.27(\mathrm{~s}, 1 \mathrm{~F})$; IR (film) $v: 3670,2983,1610,1435,1098,877,749 \mathrm{~cm}^{-1}$; MS (ESI) $m / z: 281[\mathrm{M}+\mathrm{H}]^{+}$; HRMS (ESI-TOF) calcd for $\mathrm{C}_{11} \mathrm{H}_{9} \mathrm{~F}_{4} \mathrm{O}_{2} \mathrm{~S}[\mathrm{M}+\mathrm{H}]^{+}: 281.0254$, found 281.0256.

(E)-4-(4-Chlorophenyl)-4-hydroxy-3-(trifluoromethylthio)but-3-en-2-one (2i): yellow oil, $88.7 \mathrm{mg}, 60 \%$ yield. ${ }^{1} \mathrm{H}$ NMR (400 MHz, $\left.\mathrm{CDCl}_{3}\right) \delta: 7.50(\mathrm{~d}, J=8.6 \mathrm{~Hz}, 2 \mathrm{H})$, $7.33(\mathrm{~d}, J=8.6 \mathrm{~Hz}, 2 \mathrm{H}), 2.48(\mathrm{~s}, 3 \mathrm{H}) ;{ }^{13} \mathrm{C} \mathrm{NMR}(101 \mathrm{MHz}$, $\left.\mathrm{CDCl}_{3}\right) \delta: 200.5,192.9,136.6,133.0,129.2,128.0(\mathrm{~s}, J=$ $312.1 \mathrm{~Hz}), 127.2,94.2,24.1 ;{ }^{19} \mathrm{~F}$ NMR (376 $\mathrm{MHz}, \mathrm{CDCl}_{3}$ ) $\delta:-45.84(\mathrm{~s}, 3 \mathrm{~F})$; IR (film) $v: 3663,2978,1607,1397$, 1092, 1013, 835, $750 \mathrm{~cm}^{-1}$; MS (ESI): $m / z 297[\mathrm{M}+\mathrm{H}]^{+}$; HRMS (ESI-TOF) calcd for $\mathrm{C}_{11} \mathrm{H}_{9} \mathrm{ClF}_{3} \mathrm{O}_{2} \mathrm{~S}[\mathrm{M}+\mathrm{H}]^{+}$: 296.9958, found 296.9956.

(E)-4-(3-Chlorophenyl)-4-hydroxy-3-(trifluoromethylthio)but-3-en-2-one (2j): yellow oil, $81.4 \mathrm{mg}, 55 \%$ yield. ${ }^{1} \mathrm{H}$ NMR $\left(400 \mathrm{MHz}, \mathrm{CDCl}_{3}\right) \delta: 7.51(\mathrm{t}, J=1.9 \mathrm{~Hz}, 1 \mathrm{H})$, $7.43 \sim 7.35(\mathrm{~m}, 2 \mathrm{H}), 7.29(\mathrm{t}, J=7.9 \mathrm{~Hz}, 1 \mathrm{H}), 2.48(\mathrm{~s}, 3 \mathrm{H})$; ${ }^{13} \mathrm{C}$ NMR $\left(101 \mathrm{MHz}, \mathrm{CDCl}_{3}\right) \delta: 201.5,193.9,137.4,134.0$, $131.2,129.3,129.0(\mathrm{q}, J=312.1 \mathrm{~Hz}), 128.6,126.8,95.5$, 25.0; ${ }^{19} \mathrm{~F}$ NMR $\left(376 \mathrm{MHz}, \mathrm{CDCl}_{3}\right) \delta$ : $-45.75(\mathrm{~s}, 3 \mathrm{~F})$; IR (film) $v: 3665,2980,1514,1397,1104,888,738,552$ $\mathrm{cm}^{-1}$; MS (ESI) m/z: $297[\mathrm{M}+\mathrm{H}]^{+}$; HRMS (ESI-TOF) 
calcd for $\mathrm{C}_{11} \mathrm{H}_{9} \mathrm{ClF}_{3} \mathrm{O}_{2} \mathrm{~S}[\mathrm{M}+\mathrm{H}]^{+}$: 296.9958, found 296.9960.

(E)-4-(2-Chlorophenyl)-4-hydroxy-3-(trifluoromethylthio)but-3-en-2-one (2k): yellow oil, $55.2 \mathrm{mg}, 37 \%$ yield. ${ }^{1} \mathrm{H}$ NMR (400 MHz, $\mathrm{CDCl}_{3}$ ) $\delta: 7.34 \sim 7.23(\mathrm{~m}, 4 \mathrm{H}), 2.47$ $(\mathrm{s}, 3 \mathrm{H}) ;{ }^{13} \mathrm{C}$ NMR $\left(101 \mathrm{MHz}, \mathrm{CDCl}_{3}\right) \delta: 199.7,195.2$, $135.9,131.2,130.8,129.4,129.2$ (q, $J=312.1 \mathrm{~Hz}), 128.8$, 126.6, 97.8, 24.5; ${ }^{19} \mathrm{~F}$ NMR (376 MHz, $\left.\mathrm{CDCl}_{3}\right) \delta:-45.31$ (s, 3F); IR (film) v: 3673, 2990, 1588, 1400, 1104, 753 $\mathrm{cm}^{-1}$; MS (ESI) $\mathrm{m} / \mathrm{z}: 297[\mathrm{M}+\mathrm{H}]^{+}$; HRMS (ESI-TOF) calcd for $\mathrm{C}_{11} \mathrm{H}_{9} \mathrm{ClF}_{3} \mathrm{O}_{2} \mathrm{~S}[\mathrm{M}+\mathrm{H}]^{+}$: 296.9958, found 296.9957.

(E)-4-Hydroxy-4-(4-(trifluoromethyl)phenyl)-3-(trifluoromethylthio)but-3-en-2-one (2l): yellow oil, $68.0 \mathrm{mg}$, $41 \%$ yield. ${ }^{1} \mathrm{H}$ NMR $\left(400 \mathrm{MHz}, \mathrm{CDCl}_{3}\right) \delta: 7.66 \sim 7.61(\mathrm{~m}$, 4H), 2.52 (s, 3H); ${ }^{13} \mathrm{C}$ NMR (101 MHz, $\left.\mathrm{CDCl}_{3}\right) \delta: 200.6$, 193.1, 138.1, 131.7 (q, $J=33.3 \mathrm{~Hz}), 127.9$ (q, $J=312.1$ $\mathrm{Hz}), 127.9,124.0$ (q, $J=3.0 \mathrm{~Hz}), 122.6$ (q, $J=272.7 \mathrm{~Hz}$ ), 94.7, 24.0; ${ }^{19} \mathrm{~F}$ NMR $\left(376 \mathrm{MHz}, \mathrm{CDCl}_{3}\right) \delta$ : $-45.72(\mathrm{~s}$, 3F), -63.05 (s, 3F); IR (film) v: 3676, 2990, 2899, 1400, 1321, 1068, $889 \mathrm{~cm}^{-1}$; MS (ESI) $m / z: 331[\mathrm{M}+\mathrm{H}]^{+}$; HRMS (ESI-TOF) calcd for $\mathrm{C}_{12} \mathrm{H}_{9} \mathrm{~F}_{6} \mathrm{O}_{2} \mathrm{~S}[\mathrm{M}+\mathrm{H}]^{+}$: 331.0222, found 331.0221.

(E)-4-Hydroxy-4-(naphthalen-1-yl)-3-(trifluoromethylthio)but-3-en-2-one (2m): yellow oil, $114.3 \mathrm{mg}$, 73\% yield. ${ }^{1} \mathrm{H}$ NMR $\left(400 \mathrm{MHz}, \mathrm{CDCl}_{3}\right) \delta: 7.86(\mathrm{~d}, J=8.1 \mathrm{~Hz}, 1 \mathrm{H})$, $7.81 \sim 7.79(\mathrm{~m}, 1 \mathrm{H}), 7.67 \sim 7.65(\mathrm{~m}, 1 \mathrm{H}), 7.47 \sim 7.40(\mathrm{~m}$, $3 \mathrm{H}), 7.38 \sim 7.36(\mathrm{~m}, 1 \mathrm{H}), 2.53(\mathrm{~s}, 3 \mathrm{H}) ;{ }^{13} \mathrm{C} \mathrm{NMR}(101$ $\left.\mathrm{MHz}, \mathrm{CDCl}_{3}\right) \delta: 200.3,195.7,132.5,132.2,129.6,128.6$, 127.9 (q, $J=312.1 \mathrm{~Hz}), 127.5,126.1,125.3,125.0,123.5$, 123.4, 97.1, 24.2; ${ }^{19} \mathrm{~F}$ NMR $\left(376 \mathrm{MHz}, \mathrm{CDCl}_{3}\right) \delta:-45.48$ (s, 3F); IR (film) v: 3676, 2990, 2905, 1515, 1403, 1116, 1061, $783 \mathrm{~cm}^{-1}$; MS (ESI) $m / z: 313[\mathrm{M}+\mathrm{H}]^{+}$; HRMS (ESI-TOF) calcd for $\mathrm{C}_{15} \mathrm{H}_{12} \mathrm{~F}_{3} \mathrm{O}_{2} \mathrm{~S}[\mathrm{M}+\mathrm{H}]^{+}: 313.0505$, found 313.0503 .

(E)-4-(1-Hydroxy-3-oxo-2-(trifluoromethylthio)but-1-en yl)benzonitrile (2n): yellow oil, $52.0 \mathrm{mg}, 36 \%$ yield. ${ }^{1} \mathrm{H}$ NMR (400 MHz, $\left.\mathrm{CDCl}_{3}\right) \delta: 7.67(\mathrm{~d}, J=8.4 \mathrm{~Hz}, 2 \mathrm{H}), 7.61$ $(\mathrm{d}, J=8.4 \mathrm{~Hz}, 2 \mathrm{H}), 2.51(\mathrm{~s}, 3 \mathrm{H}) ;{ }^{13} \mathrm{C} \mathrm{NMR}(101 \mathrm{MHz}$, $\mathrm{CDCl}_{3}$ ) $\delta: 200.6,192.8,138.8,130.8,128.1,127.8$ (q, $J=$ $312.1 \mathrm{~Hz}), 116.9,113.7,94.7,23.9 ;{ }^{19} \mathrm{~F}$ NMR $(376 \mathrm{MHz}$, $\left.\mathrm{CDCl}_{3}\right) \delta:-45.68(\mathrm{~s}, 3 \mathrm{~F})$; IR (film) v: 3672, 2928, 2231, 1530, 1401, 1105, 840, $732 \mathrm{~cm}^{-1}$; MS (ESI) $\mathrm{m} / \mathrm{z}: 288$ $[\mathrm{M}+\mathrm{H}]^{+}$; HRMS (ESI-TOF) calcd for $\mathrm{C}_{12} \mathrm{H}_{9} \mathrm{~F}_{3} \mathrm{NO}_{2} \mathrm{~S}$ $[\mathrm{M}+\mathrm{H}]^{+}:$: 288.0301, found 288.0298.

(E)-4-Hydroxy-4-(4-nitrophenyl)-3-(trifluoromethylthio)but-3-en-2-one (2o): yellow oil, $40.3 \mathrm{mg}, 26 \%$ yield. ${ }^{1} \mathrm{H}$ NMR (400 MHz, $\left.\mathrm{CDCl}_{3}\right) \delta: 8.22(\mathrm{~d}, J=8.2 \mathrm{~Hz}, 2 \mathrm{H}), 7.67$ $(\mathrm{d}, J=7.9 \mathrm{~Hz}, 2 \mathrm{H}), 2.51(\mathrm{~s}, 3 \mathrm{H}) ;{ }^{13} \mathrm{C} \mathrm{NMR}(101 \mathrm{MHz}$, $\left.\mathrm{CDCl}_{3}\right) \delta 200.6,192.7,148.0,140.6,128.5,127.8(\mathrm{q}, J=$ $313.1 \mathrm{~Hz}), 122.2,94.8,23.9 ;{ }^{19} \mathrm{~F}$ NMR $\left(376 \mathrm{MHz} \mathrm{CDCl}_{3}\right)$ $\delta:-45.68(\mathrm{~s}, 3 \mathrm{~F})$; IR (film) $v: 3673,2996,2899,1527$, 1401, 1107, 862, 832, $708 \mathrm{~cm}^{-1}$; MS (ESI) m/z: $308[\mathrm{M}+$ $\mathrm{H}]^{+}$; HRMS (ESI-TOF) calcd for $\mathrm{C}_{11} \mathrm{H}_{9} \mathrm{~F}_{3} \mathrm{NO}_{4} \mathrm{~S}[\mathrm{M}+\mathrm{H}]^{+}$: 308.0199, found 308.0199.

(E)-4-Hydroxy-4-(thiophen-2-yl)-3-(trifluoromethylthio)but-3-en-2-one (2p): yellow oil, $76.9 \mathrm{mg}, 57 \%$ yield. ${ }^{1} \mathrm{H}$
NMR (400 MHz, $\left.\mathrm{CDCl}_{3}\right) \delta: 8.23(\mathrm{dd}, J=4.0,1.3 \mathrm{~Hz}, 1 \mathrm{H})$, $7.64(\mathrm{dd}, J=5.0,1.3 \mathrm{~Hz}, 1 \mathrm{H}), 7.07(\mathrm{dd}, J=5.0,4.0 \mathrm{~Hz}$, 1H), 2.47 (s, 3H); ${ }^{13} \mathrm{C}$ NMR (101 MHz, $\left.\mathrm{CDCl}_{3}\right) \delta: 201.4$, $183.9,137.5,136.6,135.7,129.1$ (q, $J=313.1 \mathrm{~Hz}), 127.6$, 92.8, 25.2; ${ }^{19} \mathrm{~F}$ NMR $\left(376 \mathrm{MHz}, \mathrm{CDCl}_{3}\right) \delta:-45.23(\mathrm{~s}$, 3F); IR (film) v: 3674, 2993, 1513, 1408, 1096, 858, 718, $562 \mathrm{~cm}^{-1}$; MS (ESI): $\mathrm{m} / z 269[\mathrm{M}+\mathrm{H}]^{+}$; HRMS (ESITOF) calcd for $\mathrm{C}_{9} \mathrm{H}_{8} \mathrm{~F}_{3} \mathrm{O}_{2} \mathrm{~S}_{2}[\mathrm{M}+\mathrm{H}]^{+}:$268.9912, found 268.9910 .

(E)-1-Hydroxy-1-phenyl-2-(trifluoromethylthio)pent-1en-3-one (2q): yellow oil, $84.6 \mathrm{mg}, 61 \%$ yield. ${ }^{1} \mathrm{H}$ NMR $\left(400 \mathrm{MHz}, \mathrm{CDCl}_{3}\right) \delta: 7.57 \sim 7.50(\mathrm{~m}, 2 \mathrm{H}), 7.41(\mathrm{~d}, J=7.3$ $\mathrm{Hz}, 1 \mathrm{H}), 7.37 \sim 7.33(\mathrm{~m}, 2 \mathrm{H}), 2.97 \sim 2.83(\mathrm{~m}, 2 \mathrm{H}), 1.15(\mathrm{t}$, $J=7.4 \mathrm{~Hz}, 3 \mathrm{H}) ;{ }^{13} \mathrm{C}$ NMR $\left(101 \mathrm{MHz}, \mathrm{CDCl}_{3}\right) \delta: 204.5$, $192.9,134.5,130.1,128.0$ (q, $J=312.1 \mathrm{~Hz}$ ), 127.7, 126.8, 93.8, 29.9, 8.1; ${ }^{19} \mathrm{~F}$ NMR $\left(376 \mathrm{MHz}, \mathrm{CDCl}_{3}\right) \delta:-45.79$ (s, 3F); IR (film) v: 3673, 2984, 1530, 1397, 1100, 692, $543 \mathrm{~cm}^{-1}$; MS (ESI) $m / z: 277[\mathrm{M}+\mathrm{H}]^{+}$; HRMS (ESI-TOF) calcd for $\mathrm{C}_{12} \mathrm{H}_{12} \mathrm{~F}_{3} \mathrm{O}_{2} \mathrm{~S}[\mathrm{M}+\mathrm{H}]^{+}:$277.0505, found 277.0507 .

Supporting Information Preparation of substrates. ${ }^{1} \mathrm{H}$ NMR, ${ }^{13} \mathrm{C}$ NMR, and ${ }^{19} \mathrm{~F}$ NMR spectra for all new compounds. The Supporting Information is available free of charge via the Internet at http://sioc-journal.cn/.

\section{References}

[1] (a) Purser, S.; Moore, P. R.; Swallow, S.; Gouverneur, V. Chem. Soc. Rev. 2008, 37, 320.

(b) Meanwell, N. A. J. Med. Chem. 2011, 54, 2529.

(c) Cametti, M.; Crousse, B.; Metrangolo, P.; Milani, R.; Resnati, G. Chem. Soc. Rev. 2012, 41, 31.

(d) Wang, J.; Sánchez-Roselló, M.; Aceña, J. L.; del Pozo, C.; Sorochinsky, A. E.; Fustero, S.; Soloshonok, V. A.; Liu, H. Chem. Rev. 2014, 114, 2432.

(e) Gouverneur, V.; Seppelt, K. Chem. Rev. 2015, 115, 563.

(f) Zhou, Y.; Wang, J.; Gu, Z.; Wang, S.; Zhu, W.; Aceña, J. L.; Soloshonok, V. A.; Izawa, K.; Liu, H. Chem. Rev. 2016, 116, 422.

(g) Rong, J.; Ni, C.; Wang, Y.; Kuang, C.; Gu, Y.; Hu, J. Acta Chim. Sinica 2017, 75, 105 (in Chinese).

(荣健, 倪传法, 王云泽, 匡翠文, 顾玉诚, 胡金波, 化学学报, 2017, 75, 105.)

(h) Meanwell, N. A. J. Med. Chem. 2018, 61, 5822.

[2] (a) Hansch, C.; Leo, A.; Unger, S. H.; Kim, K. H.; Nikaitani, D.; Lien, E. J. J. Med. Chem. 1973, 16, 1207.

(b) Hansch, C.; Leo, A.; Taft, R. W. Chem. Rev. 1991, 91, 165

[3] (a) Chu, L.; Qing, F.-L. Acc. Chem. Res. 2014, 47, 1513.

(b) Toulgoat, F.; Alazet, S.; Billard, T. Eur. J. Org. Chem. 2014, 2415.

(c) Shao, X.; Xu, C.; Lu, L.; Shen, Q. Acc. Chem. Res. 2015, 48, 1227.

(d) Xu, H.-H.; Matsuzaki, K.; Shibata, N. Chem. Rev. 2015, 115, 731.

(e) Zhang, K.; Xu, X.-H.; Qing, F.-L. Chin. J. Org. Chem. 2015, 35, 556 (in Chinese).

(张珂, 徐修华, 卿凤翎, 有机化学, 2015, 35, 556.)

(f) Chachignon, H.; Cahard, D. Chin. J. Chem. 2016, 34, 445.

(g) Barata-Vallejo, S.; Bonesi, S.; Postigo, A. Org. Biomol. Chem. 2016, 14, 7150 .

(h) Zheng, H.; Huang, Y.; Weng, Z. Tetrahedron Lett. 2016, 57, 1397.

[4] (a) Sheng, J.; Li, S.; Wu, J. Chem. Commun. 2014, 50, 578. 
(b) Zhang, K.; Liu, J.-B.; Qing, F.-L. Chem. Commun. 2014, 50, 14157.

(c) Zhu, L.; Wang, G.; Guo, Q.; Xu, Z.; Zhang, D.; Wang, R. Org. Lett. 2014, 16, 5390

(d) Yang, T.; Lu, L.; Shen, Q. Chem. Commun. 2015, 51, 5479.

(e) Chen, D.-Q.; Gao, P.; Zhou, P.-X.; Song, X.-R.; Qiu, Y.-F.; Liu, X.-Y.; Liang, Y. M. Chem. Commun. 2015, 51, 6637.

(f) Fuentes, N.; Kong, W.; Fernández-Sánchez, L.; Merino, E.; Nevado, C. J. Am. Chem. Soc. 2015, 137, 964.

(g) Luo, J.; Zhu, Z.; Liu, Y.; Zhao, X. Org. Lett. 2015, 17, 3620.

(h) Xu, C.; Shen, Q. Org. Lett. 2015, 17, 4561.

(i) Liu, X.; An, R.; Zhang, X.; Luo, J.; Zhao, X. Angew. Chem., Int. Ed. 2016, 55, 5846.

(j) Zhang, P.; Li, M.; Xue, X.-S.; Xu, C.; Zhao, Q.; Liu, Y.; Wang, H.; Guo, Y.; Lu, L.; Shen, Q. J. Org. Chem. 2016, 81, 7486.

(k) Jin, D.-P.; Gao, P.; Chen, D.-Q.; Chen, S.; Wang, J.; Liu, X.-Y.; Liang, Y.-M. Org. Lett. 2016, 18, 3486.

(1) Luo, J.; Liu, Y.; Zhao, X. Org. Lett. 2017, 19, 3434.

(m) Pan, S.; Huang, Y.; Xu, X.-H.; Qing, F.-L. Org. Lett. 2017, 19, 4624.

(n) Liu, X.; Liang, Y.; Ji, J.; Luo, J.; Zhao, X. J. Am. Chem. Soc. 2018, 140, 4782.

(o) Xiao, Z.; Liu, Y.; Zheng, L.; Liu, C.; Guo, Y.; Chen, Q.-Y. J. Org. Chem. 2018, 83, 5836.

(p) Xi, C.-C.; Chen, Z.-M.; Zhang, S.-Y.; Tu, Y.-Q. Org. Lett. 2018, 20, 4227.

[5] Wu, W.; Dai, W.; Ji, X.; Cao, S. Org. Lett. 2016, 18, 2918.

[6] (a) Guo, C.-H.; Chen, D.-Q.; Chen, S.; Liu, X.-Y. Adv. Synth. Catal. 2017, 359, 2901.

(b) Li, H.; Liu, S.; Huang, Y.; Xu, X.-H.; Qing, F.-L. Chem. Commun. 2017, 53, 10136.

[7] (a) Tlili, A.; Alazet, S.; Glenadel, Q.; Billard, T. Chem.-Eur. J. 2016, 22, 10230.

(b) Pan, S.; Li, H.; Huang, Y.; Xu, X.-H.; Qing, F.-L. Org. Lett. 2017, 19, 3247

[8] Jiang, L.; Ding, T.; Yi, W.-B.; Zeng, X.; Zhang, W. Org. Lett. 2018, 20, 2236

[9] (a) Ferry, A.; Billard, T.; Langlois, B. R.; Bacqué, E. Angew. Chem., Int. Ed. 2009, 48, 8551.

(b) Wu, J.-J.; Xu, J.; Zhao, X. Chem.-Eur. J. 2016, 22, 15265.

[10] (a) Yang, Y.; Jiang, X.-L.; Qing, F.-L. J. Org. Chem. 2012, 77, 7538 .

(b) Wu, X.; Chu, L.; Qing, F.-L. Angew. Chem. Int. Ed. 2013, 52, 2198.

(c) Jiang, X.-Y.; Qing, F.-L. Angew. Chem. Int. Ed. 2013, 52, 14177.

(d) Yu, W.; Xu, X.-H.; Qing, F.-L. Adv. Synth. Catal. 2015, 357,
2039.

(e) Yang, B.; Xu, X.-H.; Qing, F.-L. Org. Lett. 2015, 17, 1906

(f) Lin, Q.-Y.; Xu, X.-H.; Zhang, K.; Qing, F.-L. Angew. Chem., Int. Ed. 2016, 55, 1479.

(g) Yang, B.; Xu, X.-H.; Qing, F.-L. Chin. J. Chem. 2016, 34, 465.

(h) Lin, Q.-Y.; Ran, Y.; Xu, X.-H.; Qing, F.-L. Org. Lett. 2016, 18, 2419.

(i) Yu, W.; Xu, X.-H.; Qing, F.-L. Org. Lett. 2016, 18, 5130.

(j) Yang, B.; Xu, X.-H.; Qing, F.-L. Org. Lett. 2016, 18, 5956.

(k) Yang, B.; Yu, D.; Xu, X.-H.; Qing, F.-L. ACS Catal. 2018, 8 , 2839.

(1) Ouyang, Y.; Xu, X.-H.; Qing, F.-L. Angew. Chem., Int. Ed. 2018 , 57,6926 .

[11] (a) Yin, F.; Wang, X.-S. Org. Lett. 2014, 16, 1128

(b) Guo, S.; Zhang, X.; Tang, P. Angew. Chem., Int. Ed. 2015, 54, 4065 .

(c) Wu, H.; Xiao, Z.; Wu, J.; Guo, Y.; Xiao, J.-C.; Liu, C.; Chen, Q.-Y. Angew. Chem., Int. Ed. 2015, 54, 4070.

(d) Qiu, Y.-F.; Zhu, X.-Y.; Li, Y.-X.; He, Y.-T.; Yang, F.; Wang, J.; Hua, H.-L.; Zheng, L.; Wang, L.-C.; Liu, X.-Y.; Liang, Y.-M. Org. Lett. 2015, 17, 3694.

(e) Zeng, Y.-F.; Tan, D.-H.; Chen, Y.; Lv, W.-X.; Liu, X.-G.; Li, Q.; Wang, H. Org. Chem. Front. 2015, 2, 1511.

(f) Li, C.; Zhang, K.; Xu, X.-H.; Qing, F.-L. Tetrahedron Lett. 2015 , $56,6273$.

(g) Pan, S.; Huang, Y.; Qing, F. L. Chem. Asian J. 2016, 11, 2854.

(h) Huang, F.-Q.; Wang, Y.-W.; Sun, J.-G.; Xie, J.; Qi, L.-W.; Zhang, B. RSC Adv. 2016, 6, 52710.

(i) Song, Y.-K.; Qian, P.-C.; Chen, F.; Deng, C.-L.; Zhang, X.-G. Tetrahedron 2016, 72, 7589 .

(j) Ji, M. S.; Wu, Z.; Yu, J. J.; Wan, X. B.; Zhu, C. Adv. Synth. Catal. 2017, 359, 1959.

(k) He, B.; Xiao, Z.; Wu, H.; Guo, Y.; Chen, Q.-Y.; Liu, C. RSC Adv. 2017, 7, 880 .

(1) Liu, K.; Jin, Q.; Chen, S.; Liu, P. N. RSC Adv. 2017, 7, 1546.

(m) Li, M.; Petersen, J. L.; Hoover, J. M. Org. Lett. 2017, 19, 638.

(n) Cheng, Z.-F.; Tao, T.-T.; Feng, Y.-S.; Tang, W.-K.; Xu, J.; Dai, J.-J.; Xu, H.-J. J. Org. Chem. 2018, 83, 499.

[12] (a) Ye, Y.; Sanford, M. S. J. Am. Chem. Soc. 2012, 134, 9034.

(b) Huang, L.; Zheng, S.-C.; Tan, B.; Liu, X.-Y. Chem.-Eur. J. 2015, 18, 6718 .

(c) Yu, L.-Z.; Wei, Y.; Shi, M. Chem. Commun. 2016, 52, 13163.

(d) Cheng, C.; Liu, S.; Lu, D.; Zhu, G. Org. Lett. 2016, 18, 2852.

[13] (a) Wu, W.; Zhang, X.; Liang, F.; Cao, S. Org. Biomol. Chem. 2015, 13, 6992 .

(b) Zhang, J.; Yang, J.-D.; Zheng, H.; Xue, X.-S.; Mayr, H.; Cheng, J.-P. Angew. Chem. Int. Ed. 2018, 57, 12690.

(Li, L.; Fan, Y.) 